\title{
Observational Constraints on the Completeness of Space near Astrophysical Objects
}

\author{
Timothy Clifton ${ }^{1 *}$ and John D. Barrow ${ }^{2 \dagger}$ \\ ${ }^{1}$ Department of Astrophysics, University of Oxford, UK. \\ ${ }^{2}$ DAMTP, University of Cambridge, UK.
}

(Dated: March 10, 2010)

\begin{abstract}
We consider the observational effects of a deficit angle, $w$, in the topology of the solar system and in the 'double pulsar' system PSR J0737-3039A/B. Using observations of the perihelion precession of Mercury, and the gravitational deflection of light due to the Sun, we constrain the magnitude of such a deficit angle in the solar system to be $2 \pi(1-w)$, with $0 \leq(1-w)<10^{-9}$ at $95 \%$ confidence. We calculate the effects of a deficit angle on the periastron advance, geodetic precession rate and inclination angle of the double pulsar system and use the observational data to obtain the constraint $0 \leq(1-w)<2.4 \times 10^{-8}$ at $95 \%$ confidence. Although this result is weaker than the solar system bound, it is in a very different physical environment, where accumulating data is likely to lead to tighter constraints in the future.
\end{abstract}

PACS numbers: 95.30.Sf, 97.60.Gb

\section{INTRODUCTION}

Metric-based gravity theories, like Einstein's and its close relatives, are routinely tested using observations of astrophysical systems where gravitational fields are strong and non-Newtonian. Following Eddington's introduction of a parameterised form of the metric in 1922 1], and Nordvedt's extension to more general configurations by including further parameters 2], a sophisticated frame-work has been devised [3], and a large number of careful observations made, in order to place stringent constraints on the geometry of space-time. However, most of these studies have assumed that the astrophysical systems they consider should have a trivial space-time topology. Under such an assumption, it is possible to make strong statements about the magnitude of any deviations from the predictions of general relativity. Here we take a different approach: We assume a geometry that is locally isometric to the predictions of general relativity, but which permits non-trivial global topologies. The astrophysical observations can then be used to constrain the topology of the systems in question. Of particular interest for this study is the recently discovered 'double pulsar' system, PSR J0737-3039A/B [4, 5].

Non-trivial topologies can exist in a number of astrophysically interesting situations, including magnetic monopoles, cosmic strings, domain walls and textures [ 6 ], all of which can arise in phase transitions in the early universe [7]. A tell-tale sign of such topological defects is the existence of a 'deficit angle', whereby a wedge of space-time appears to have been removed, and the surfaces that remain have been identified. A simple example of this feature, first found by Marder in 1959 [8], is the

\footnotetext{
*tclifton@astro.ox.ac.uk

${ }_{\dagger}^{\dagger}$ j.d.barrow@damtp.cam.ac.uk
}

line-element

$$
d s^{2}=-\alpha d t^{2}+\frac{d r^{2}}{\alpha}+r^{2} d \theta^{2}+w^{2} r^{2} \sin ^{2} \theta d \phi^{2},
$$

where $\alpha=1-2 G M / r$ and $w \in(0,1]$ is a constant. This is well known as the exact solution of a point-like mass on an infinitely thin cosmic string [8, 9]. It is clearly locally isometric to the Schwarzschild solution, but if we allow $\phi$ to run from 0 to $2 \pi$, then we see it has a different topology.

The line-element (1) may initially appear a trivial manipulation of the Schwarzschild solution. The effect of introducing such a topological defect, however, has nontrivial consequences for the geodesic equations. For the metric above, these equations have recently been solved completely by Hackmann et al. [10]. The missing wedge of space-time causes new behaviour that is not present in the Schwarzschild solution, such as a precession of the angular momentum vector about the axis of the string 11]. By using observational constraints on such phenomena we can therefore constrain the amplitude of any possible deficit angle, and hence place constraints on the topologies of astrophysical systems.

In Section [I] we summarise how deficit angles affect the geodesic equations, and observations of gravitational phenomena in the solar system. in Section [III we proceed to consider the double pulsar. This over-constrained system has a large number of well measured observables, and provides an excellent laboratory to perform the type of test we are considering. In Section IV] we give our conclusions.

\section{SOLAR SYSTEM CONSTRAINTS}

If we choose coordinates so that the orbit we consider is in a plane of constant elevation $(\theta=\pi / 2)$, then the effect of a deficit angle on the equations of motion enters only through the azimuthal coordinate, $\phi$. To include 
such a defect, we can then write the range of $\phi$ as

$$
0 \leq \phi \leq 2 \pi-\Delta \varphi
$$

where $\Delta \varphi \in[0,2 \pi)$ is the deficit angle. Alternatively, we can change variables so that $\Delta \varphi=2 \pi(1-w)$. Now, $\phi$ covers the range

$$
0 \leq \phi \leq 2 \pi w
$$

which is equivalent to a simple coordinate redefinition

$$
\phi \rightarrow w \phi
$$

\section{A. Time-like orbits}

Using the redefinition (4), the geodesic equations become

$$
u^{\prime \prime}+w^{2} u=\frac{G M}{L^{2}}+3 G M w^{2} u^{2}
$$

where $u=1 / r$, and where primes denote differentiation with respect to $\phi$. The angular momentum constant, $L$, is given by

$$
L=r^{2} \sin \left(\theta_{0}\right) \frac{d \phi}{d \lambda},
$$

where $\lambda$ parameterises distance along the curve. The solution to (5) is then given by

$$
u=w^{-2} u_{G R}(w \phi)
$$

where $u_{G R}(\phi)$ is the usual general relativistic solution that occurs when $w=1$. Therefore, we have

$$
u=\frac{1}{r}=\frac{G M}{w^{2} L^{2}}\left[1+e \cos \left\{w\left(\phi-\phi_{0}-\Delta \phi_{0}\right)\right\}\right]
$$

where $e$ is the eccentricity of the orbit, $\phi_{0}$ specifies the phase, and $\Delta \phi_{0}$ gives the correction due to relativistic perihelion precession.

It can now be seen from (8) that the perihelion shift per orbital revolution is

$$
w \Delta \phi=\frac{6 \pi G^{2} M^{2}}{w^{2} L^{2}}+2 \pi(1-w) .
$$

If we now write

$$
w=1-\delta w
$$

where $\delta w \in[0,1)$ is small, then to first order we should expect the precession to be

$$
\Delta \phi \simeq \Delta \phi_{G R}+2 \pi \delta w
$$

where $\Delta \phi_{G R}=\{\Delta \phi\}_{w=1}$ is just the usual general relativistic prediction with no defect. The precision of the agreement with the current data therefore gives a bound on $\delta w$, and hence on $w$.
In order to gain observations of $\Delta \phi$ from the precession of the orbit of Mercury, with respect to the vernal equinox of the Sun, it is necessary to take into account the precession of the equinoxes on the coordinate system (about 5025" per century), the perturbing effects of the other planets (about 531" per century) and the effect of the quadrupole moment of the Sun (about 0.025" per century), on the perihelion precession of the objects that orbit it. These challenges can be addressed in a variety of different ways, and the result is authors claiming slightly different observational bounds on the residual $\Delta \phi$. Rather than favoring any particular method here, we prefer to quote a number of different precise calculations, obtained by various authors. These are displayed in Table 1. For further details the reader is referred to the original papers, and references therein. For an overview of the issues involved the reader is referred to [18].

\begin{tabular}{|c|c|c|}
\hline Source & $\begin{array}{c}\Delta \phi-\Delta \phi_{G R} \\
(\operatorname{arcsec} / \text { century })\end{array}$ & $\begin{array}{c}10^{10} \times \delta w \\
(2 \sigma \text { upper bound })\end{array}$ \\
\hline Anderson et al. [14] & $-0.04 \pm 0.20$ & 6.7 \\
Anderson et al. [15] & $+0.15 \pm 0.14$ & 8.0 \\
Krasinsky et al. [16] & & \\
EPM1988 & $+0.004 \pm 0.061$ & 2.3 \\
DE200 & $-0.003 \pm 0.061$ & 2.2 \\
Pitjeva [17] & & \\
EPM1988 & $-0.017 \pm 0.052$ & 1.6 \\
DE200 & $-0.011 \pm 0.052$ & 1.7 \\
\hline
\end{tabular}

TABLE I: The value of the perihelion precession of Mercury obtained from observations by various authors, and the resulting $2 \sigma$ upper bound on the permissible values of $\delta w$. The acronyms EPM1988 and DE200 refer to different numerical ephemerides, which are reviewed in [12]. We take the sidereal period of Mercury to be 0.24 years [13].

It can be seen from Table \ that placing a constraint on $\delta w$ is not a clear-cut matter, and depends both on the data used, and how it is treated. However, all of the results in Table II are consistent with the conservative statement that the $2 \sigma$ bound on $\delta w$ from observations of the perihelion precession of Mercury is within the range

$$
0 \leq \delta w<10^{-9}
$$

\section{B. Null orbits}

The geodesic equation for light rays is transformed in a similar way, but there is now no $L$. Under the redefinition (4), we therefore have simply

$$
u^{\prime \prime}+w^{2} u=3 G M w^{2} u^{2},
$$

which has the solution

$$
u=u_{G R}(w \phi),
$$


where, again, $u_{G R}(\phi)$ is the usual general relativistic solution with $w=1$. Taking this, together with $\phi=\pi+\delta \phi$, we obtain

$$
u=\frac{1}{r}=\frac{4 G M}{R^{2}}-\frac{w \delta \phi}{R}+\frac{\pi}{R}(1-w),
$$

where $R$ is the impact parameter. The total deflection is then

$$
w \delta \phi=\frac{4 G M}{R}+\pi(1-w)=\frac{4 G M}{R}+\pi \delta w,
$$

and the deflection caused by the defect is

$$
\delta \phi \simeq \delta \phi_{G R}+\pi \delta w,
$$

where $\delta \phi_{G R}=\{\delta \phi\}_{w=1}$ is the usual general relativistic prediction. The bound on $\delta w$, and hence $w$, can now be obtained by comparing (17) with observed deflections.

The best results available on light bending in gravitational fields are those of Shapiro, Davis, Lebach and Gregory [19]. These authors use almost 2 million observations of 541 radio sources by 87 Very-Long-Baseline Interferometry (VLBI) sites to calculate the deflection caused by the gravitational field of the Sun. Their result is

$$
\delta \phi=(0.99992 \pm 0.00023) \delta \phi_{G R},
$$

which gives us a $2 \sigma$ bound on $\delta w$ of

$$
0 \leq \delta w<9.9 \times 10^{-10},
$$

where we have taken the general relativistic prediction of light bending for an object whose light ray grazes the Sun's limb to be $1.75^{\prime \prime}$. This is almost exactly the same bound as was achieved for the time-like case [27].

\section{PULSAR CONSTRAINTS}

The recent discovery of the 'double pulsar' system [4, 5], PSR J0737-3039A/B, provides new opportunities for testing ideas in relativistic gravity [20, 21]. These binary neutron stars are known to orbit each other with a period of 2.45 hours, with much higher velocities and accelerations than those found in other binary pulsar systems. By good fortune, this system is also relatively near to the Sun, and oriented so that we observe it nearly edge-on, at an inclination angle of about $89^{\circ}$. Most importantly, however, and unique to PSR J0737-3039A/B, is the feature that both neutron stars are detectable as radio pulsars, with periods of $22 \mathrm{~ms}$ and 2.7s, respectively for PSR J0737-3039A and PSR J07373039B. These properties make the double pulsar an excellent tool for constraining the type of deviant gravitational phenomena we are considering in this article.

To proceed further, we model the space-time geometry as

$$
d s^{2}=-(1-2 U) d t^{2}+(1+2 U) \tilde{\delta}_{i j} d x^{i} d x^{j}
$$

where $\tilde{\delta}_{i j}$ specifies the static geometry of the 3 -space. We can now assign orders of smallness in the usual way, so that the Newtonian potential $U$ is $O(2)$ small, time derivatives add an $O(1)$ of smallness, and the 3-velocity $v^{i}$ is $O(1)$ small.

We will now consider relativistic effects that are good candidates for constraining the existence of any deficit angle in the double pulsar, before comparing our predictions with the observational data.

\section{A. Spin Precession}

Geodetic precession of a body's spin vector about its orbital angular momentum vector is a well known prediction of relativistic gravity, and has already been studied in some binary pulsar systems [21 26]. However, while it took over a decade for geodetic precession to be observed in the Hulse-Taylor pulsar, PSR B1913+16 [23], it has already been reported in the double pulsar [21]. We expect this effect to be particularly sensitive to the existence of a deficit angle, as it involves integration over an orbit. We shall therefore calculate its influence below, before proceeding to infer constraints from observational data later in the section.

First, we want the pulsar spin vector, $Y^{\mu}$, to be orthogonal to the world-line of a particle, $u^{\mu}$, and to be parallel propagated along that curve, so that

$$
\frac{d Y_{\mu}}{d \tau}=g^{\lambda \sigma} \Gamma_{\sigma \mu \nu} Y_{\lambda} \frac{d x^{\nu}}{d \tau}=\Gamma_{\sigma \mu \nu} Y^{\sigma} \frac{d x^{\nu}}{d \tau},
$$

where $\Gamma_{\mu \nu \sigma}=g_{\mu \rho} \Gamma_{\nu \sigma}^{\rho}$, and $\tau$ is proper time along the particle's world-line. The orthogonality condition then gives

$$
Y_{0}=-v^{i} Y_{i}+O(3),
$$

where $v^{i}$ is the 3 -velocity of the particle. Multiplying through by $d \tau / d t$ we find that the spatial component of $Y^{\mu}$ obeys

$$
\begin{aligned}
\frac{d Y_{i}}{d t}= & \Gamma_{0 i 0} v^{j} Y_{j}+\Gamma_{j i 0} Y^{j}+\Gamma_{k i j} v^{j} Y^{k}+O(5) \\
= & \tilde{\delta}_{i j} U_{, 0} Y^{j}+\left(2 \tilde{\delta}_{k j} U_{, i}+\tilde{\delta}_{i k} U_{, j}-\tilde{\delta}_{i j} U_{, k}\right) v^{j} Y^{k} \\
& +(1+2 U) \tilde{\Gamma}_{k i j} v^{j} Y^{k}+g_{0[k, i]} Y^{k}+O(5),
\end{aligned}
$$

where $\tilde{\Gamma}_{i j k}=\frac{1}{2}\left(\tilde{\delta}_{i j, k}+\tilde{\delta}_{i k, j}-\tilde{\delta}_{j k, i}\right)$. The magnitude of the 4-vector $Y^{\mu}$ should remain constant along the curve specified by $u^{\mu}$, so that

$$
\frac{d}{d \tau}\left(g^{\mu \nu} Y_{\mu} Y_{\nu}\right)=0
$$

or, up to $O(2)$,

$$
-\left(v^{i} Y_{i}\right)^{2}+(1-2 U) \tilde{\delta}^{i j} Y_{i} Y_{j}=\text { constant. }
$$

This implies that a spin 3-vector, $S^{i}$, with constant magnitude, $\tilde{\delta}^{i j} S_{i} S_{j}$, is given by

$$
Y_{i}=(1+U) S_{i}+\frac{1}{2} \tilde{\delta}_{i j} v^{j} v^{k} S_{k}+O(4) .
$$


Alternatively, inverting this expression gives

$$
\begin{aligned}
S_{i} & =(1-U) Y_{i}-\frac{1}{2} \tilde{\delta}_{i j} v^{j} v^{k} Y_{k}+O(4) \\
& =(1+U) \tilde{\delta}_{i j} Y^{j}-\frac{1}{2} \tilde{\delta}_{i j} \tilde{\delta}_{k m} v^{j} v^{k} Y^{m}+O(4),
\end{aligned}
$$

which can now be differentiated with respect to $t$, and $d Y_{i} / d t$ can be substituted from above, to give

$$
\frac{d S_{i}}{d t}=\left[3 v^{j} \tilde{\delta}^{k m} \tilde{\delta}_{j[k} U_{, i]}+\tilde{\delta}^{k m} g_{0[k, i]}+\tilde{\Gamma}_{i j}^{m} v^{j}\right] S_{m}+O(4) .
$$

where we have used

$$
\frac{d v^{i}}{d t}=\tilde{\delta}^{i j} U_{j}-\tilde{\Gamma}_{j k}^{i} v^{j} v^{k}
$$

For shorthand, one can also write

$$
\hat{\Omega}_{k i} \equiv 3 v^{j} \tilde{\delta}_{j[k} U_{, i]}+g_{0[k, i]}
$$

and

$$
\Omega^{j} \equiv \frac{1}{2} \epsilon^{j k i} \hat{\Omega}_{k i}
$$

so that

$$
\frac{d S_{i}}{d t}=\left[\Omega^{j} \epsilon_{j k i} \tilde{\delta}^{k m}+\tilde{\Gamma}_{i j}^{m} v^{j}\right] S_{m}+O(4) .
$$

This is the general expression for spin precession in the geometry (20).

To see the effect of a deficit angle on spin precession we can now consider a 3-metric that is locally isometric to Euclidean 3-space under the coordinate redefinition (4). For an elliptic orbit in a plane of constant $\theta$, and with a potential of the form $U=U(r)$, we then have $v^{\theta}=0$ and $U_{, \theta}=U_{, \phi}=0$. From (33), we then find

$$
\begin{aligned}
\frac{d S_{r}}{d t} & =\frac{3}{2} v^{\phi} U_{, r} S_{\phi}+\frac{v^{\phi}}{r} S_{\phi} \\
\frac{d S_{\phi}}{d t} & =-\frac{3}{2} v^{\phi} r^{2} w^{2} U_{, r} S_{r}-r w^{2} v^{\phi} S_{r}+\frac{v^{r}}{r} S_{\phi} .
\end{aligned}
$$

If we now define two new quantities, $\tilde{S}_{\phi} \equiv S_{\phi} /(r w)$ and $X \equiv\left(3 v^{\phi} r U_{, r} / 2+v^{\phi}\right) w$, these equations reduce to

$$
\begin{gathered}
\frac{d S_{r}}{d t}=X \tilde{S}_{\phi} \\
\frac{d \tilde{S}_{\phi}}{d t}=-X S_{r}
\end{gathered}
$$

which have the solutions

$$
\begin{aligned}
& S_{r}=A \sin \left\{\int X d t\right\} \\
& \tilde{S}_{\phi}=A \cos \left\{\int X d t\right\}
\end{aligned}
$$

where $A$ is a constant of integration. If we now use (10), with $\delta w$ being $O(2)$ small, then we find that the precession rate integrated over an orbit is

$$
\begin{aligned}
\Omega & =2 \pi-\int X d t \\
& =2 \pi-\frac{3}{2} \int v^{\phi} r U_{, r} d t-(1-\delta w) \int v^{\phi} d t \\
& =\Omega_{G R}+2 \pi \delta w,
\end{aligned}
$$

where $\Omega_{G R}$ is the general relativistic precession rate, when $w=0$. The extra precession per orbit due to the deficit angle is therefore $2 \pi \delta w$.

This result can be seen to be straightforwardly generalisable to more complicated orbits if we note that $w$ always enters as a multiplicative factor in the $O(2)$ terms in (33). The lowest-order effect of $w$ then occurs when it multiplies the $O(0)$ terms, which are independent of gravitational potentials and velocities.

\section{B. Orbital Inclination}

Binary pulsar observations are sensitive to the sine of the inclination of the orbital plane with respect to the line of sight of the observer:

$$
s=\sin i=\frac{x}{a},
$$

where $a$ is the semi-major axis of the elliptical orbit, and $x$ is the 'projected' semi-major axis. Although the value of this quantity itself is not dependent on integration over an orbit, it is usually determined in terms of another quantity that is: The orbital period.

In the Newtonian 2-body problem the orbital period is given by

$$
P_{b}=2 \pi \sqrt{\frac{a^{3}}{m}}
$$

where $m=m_{p}+m_{c}$ is the total mass of the pulsar and its companion (another pulsar, in the case of the double pulsar). If there is a deficit angle then we should expect this period to be reduced by a factor of $w$, due to the shorter path length, so that

$$
P_{b}=2 \pi w \sqrt{\frac{a^{3}}{m}} .
$$

Using this to eliminate $a$ in (42) we then find that

$$
s=w^{2 / 3}\left(\frac{2 \pi}{P_{b}}\right)^{2 / 3} x_{p} \frac{\left(m_{p}+m_{c}\right)^{2 / 3}}{m_{c}},
$$

where we have also used $x_{p}=x m_{c} /\left(m_{p}+m_{c}\right)$, which is a directly measurable quantity. 


\section{Constraints}

The constraints on periastron advance, inclination angle and geodetic precession rate obtained in [20] and [21] for the double pulsar system are displayed in Table II. The first two of these are based on observations made between April 2003 and January 2006 using the 64m Parkes radio telescope in New South Wales, the $76 \mathrm{~m}$ Lovell radio telescope at the Jodrell Bank Observatory, and the $100 \mathrm{~m}$ Green Bank telescope in West Virginia. The observations of geodetic precession were made between December 2003 and November 2007 using the Green Bank telescope.

\begin{tabular}{|l|l|}
\hline \multicolumn{1}{|c|}{ Parameter } & \multicolumn{1}{|c|}{ Observed value } \\
\hline Periastron advance, $\dot{\omega}$ & $16.89947(68)^{\circ} /$ year \\
Orbital inclination, $s=\sin i$ & $0.99974(+16,-39)$ \\
Geodetic precession rate, $\Omega_{B}$ & $4.77(+66,-65)^{\circ} /$ year \\
\hline
\end{tabular}

TABLE II: Observational constraints on the periastron advance, $\dot{\omega}$, orbital inclination, $s=\sin i$, and geodetic precession rate of PSR J0737-3039B, $\Omega_{B}$, from [20] and 21].

Using the expressions found above, together with the usual general relativistic $(\delta w=0)$ results, we can now write down the periastron advance, inclination angle and geodetic precession rates as

$$
\begin{aligned}
& \dot{\omega}=3\left(\frac{2 \pi}{P_{b}}\right)^{5 / 3} \frac{\left(m_{p}+m_{c}\right)^{2 / 3}}{\left(1-e^{2}\right)}+\left(\frac{2 \pi}{P_{b}}\right) \delta w \\
& s=\left(1-\frac{2}{3} \delta w\right)\left(\frac{2 \pi}{P_{b}}\right)^{2 / 3} x_{p} \frac{\left(m_{p}+m_{c}\right)^{2 / 3}}{m_{c}}
\end{aligned}
$$

and

$$
\Omega_{B}=\left(\frac{2 \pi}{P_{b}}\right)^{5 / 3} \frac{1}{\left(1-e^{2}\right)} \frac{m_{c}\left(4 m_{p}+3 m_{c}\right)}{2\left(m_{p}+m_{c}\right)^{4 / 3}}+\left(\frac{2 \pi}{P_{b}}\right) \delta w .
$$

In all of these expressions $P_{b}, x_{B}$ and $e$ can be measured directly, and are given in [20]. The ratio of masses $m_{A} / m_{B}$ is also known [20], but not the individual masses themselves. We therefore need two observations to obtain a constraint on the deficit angle. The first will give enough information to find the two masses, and the second can be used to compare predicted and observed values of a relativistic effect. We choose to use $\dot{\omega}$ as the first observable in each case, as it is known to greatest accuracy.

Using the additional constraint from geodetic precession, $\Omega_{B}$, then gives the $2 \sigma$ bound [28]

$$
0 \leq \delta w<1.5 \times 10^{-6}
$$

Alternatively, we can use the inclination angle, $s$, to get the stronger $2 \sigma$ bound

$$
0 \leq \delta w<2.4 \times 10^{-8}
$$

\section{DISCUSSION}

We have considered the effects of a deficit angle, $w$, in the space-time metric for the solar system and the double pulsar system. Using observations of the perihelion precession of Mercury and the gravitational deflection of light we constrain the magnitude of such a deficit angle in the solar system to be $2 \pi(1-w)$, with

$$
0 \leq(1-w)<10^{-9}
$$

to $95 \%$ confidence. Similarly, we have used observations of periastron advance and inclination angle in the double pulsar system PSR J0737-3039A/B to gain the constraint

$$
0 \leq(1-w)<2.4 \times 10^{-8},
$$

also to $95 \%$ confidence. Although this result is weaker than the solar system bound, it is in a very different physical environment, where accuracy is likely to improve in the future, as observational data on the double pulsar accumulates.

\section{Acknowledgements}

We are grateful to Michael Kramer for helpful information. TC acknowledges the support of Jesus College, Oxford and the BIPAC.
[1] A. S. Eddington, The Mathematical Theory of Relativity, Cambridge UP, Cambridge (1922).

[2] K. Nordtvedt, Phys. Rev. 169, 1017 (1968); and Phys. Rev. 180, 1293 (1969).

[3] C. M. Will, Theory and Experiment in Gravitational Physics, rev. ed., Cambridge UP, Cambridge (1993).

[4] M. Burgay et al., Nature 426, 531 (2003).

[5] A. G. Lyne et al., Science 303, 1153 (2004).

[6] A. Vilenkin \& P. Shellard, Cosmic strings and other topological defects, Cambridge UP, Cambridge (1994).

[7] T. W. B. Kibble, J. Phys. A 9, 1387 (1976).
[8] L. Marder, Proc. Roy. Soc. A 252, 45 (1959).

[9] M. Aryal, L. Ford \& A. Vilenkin, Phys. Rev. D 34, 2263 (1986).

[10] E. Hackmann, B. Hartmann, C. Lämmerzahl \& P. Sirimachan, arXiv:0912.2327 [gr-qc] (2009).

[11] D. Gal'tsov \& E. Masar, Class. Quant. Grav. 6, 1313 (1989).

[12] E. V. Pitjeva., Celest. Mech. Dyn. Astron. 80, 249 (2001).

[13] C. W. Allen, Allen's Astrophysical Quantities, 4th edition, ed. A. N. Cox, Springer, New York (2000).

[14] J. D. Anderson et al., IAU Proceedings 9, 324 (1991). 
[15] J. D. Anderson et al., in Proceedings of the 6th Marcel Grossman Meeting on General Relativity eds. H. Sato \& T. Nakamura, World Scientific, Singapore (1992). pp. 353-355.

[16] G. A. Krasinsky et al., Celest. Mech. Dyn. Astron. 55, 1 (1993).

[17] E. V. Pitjeva, Celest. Mech. Dyn. Astron. 55, 313 (1993).

[18] S. Pireaux, J. P. Rozelot \& S. Godier, Astrophys. Sp. Sci. 284, 1159 (2003).

[19] S. S. Shapiro, J. L Davis, D. E. Lebach \& J. S. Gregory, Phys. Rev. Lett. 92, 121101 (2004).

[20] M. Kramer et al., Science 314, 97 (2006).

[21] R. P. Breton et al., Science 321, 104 (2008).

[22] J. M. Weisberg, R. W. Romani \& J. H. Taylor, Astrophys. J. 347, 1030 (1989).
[23] M. Kramer, Astrophys. J. 509, 856 (1998).

[24] J. M. Weisberg \& J. H. Taylor, Astrophys. J. 576, 942 (2002).

[25] A. W. Hotan, M. Bailes \& S. M. Ord, Astrophys. J. 624, 906 (2005).

[26] T. Clifton \& J. M. Weisberg, Astrophys. J. 679, 687 (2008).

[27] Note that these bounds are weaker than those of [10], who appear to have erroneously used constraints on postNewtonian parameters derived from the Nordvedt effect and the Shapiro effect to constrain perihelion precession and light bending.

[28] In order to combine asymmetric errors we have assumed they are Gaussian distributed on each branch, with different variances. 